DOI: $10.3901 / J M E .2020 .17 .039$

\title{
磁控微型软体爬行机器人运动特性*
}

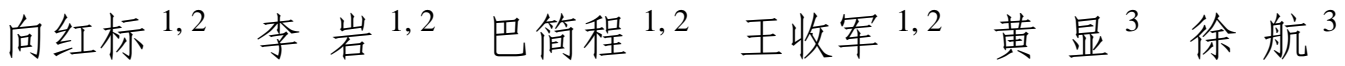 \\ (1. 天津理工大学天津市先进机电系统设计与智能控制重点实验室 天津 300384 ; \\ 2. 天津理工大学机电工程国家级实验教学示范中心 天津 300384; \\ 3. 天津大学精密仪器与光电子工程学院 天津 300372)
}

\begin{abstract}
摘要: 为实现微型机器人无缆驱动, 设计一种基于磁弹性复合材料的磁控微型软体爬行机器人, 通过三维亥姆霍兹线圈构建 变换空间磁场, 控制磁性材料产生磁力矩, 并耦合机器人重力、摩擦力及自身的弹性变形, 使得机器人实现多个连续的姿态 变换, 完成爬行动作。介绍微型软体爬行机器人结构和制作流程, 建立机器人准静态力平衡方程, 利用 Abaqus 有限元仿真 和试验对比分析了机器人弯曲变形、接触面摩擦、爬行步长、转向等运动特性, 在此基础上建立机器人爬行动作的速度模型, 研究控制信号频率与幅值对机器人爬行速度的影响, 并最终实现爬行机器人的路径规划控制。试验和仿真结果表明, 该机器 人能实现在 $x y$ 平面内任意方向的爬行动作, 为进一步揭示磁控微型机器人的运动特性及规律奠定了基础。
\end{abstract}

关键词: 无缆驱动; 微型软体机器人; 磁弹性复合材料; 爬行; 磁场控制

中图分类号: TP242

\section{Motion Characteristics of Magnetic Control Micro-soft Crawling Robot}

\author{
XIANG Hongbiao $^{1,2}$ LI Yan $^{1,2}$ BA Jiancheng ${ }^{1,2}$ WANG Shoujun ${ }^{1,2}$ \\ HUANG Xian ${ }^{3}$ XU Hang ${ }^{3}$
}

(1. Tianjin Key Laboratory for Advanced Mechatronic System Design and Intelligent Control,

Tianjin University of Technology, Tianjin 300384;

2. National Demonstration Center for Experimental Mechanical and Electrical Engineering Education,

Tianjin University of Technology, Tianjin 300384;

3. School of Precision Instruments \& Opto-Electronics Engineering, Tianjin University, Tianjin 300372)

\begin{abstract}
In order to realize the cable-less driven for the micro-robot, a magnetic control micro-soft crawling robot based on magnetic elastic composite material is designed. Variable spatial magnetic fields are set up to generate variable magnetic moment on magnetic material through 3D Helmholtz coils. Coupled with the magnetic moment, gravity, friction and elastic deformation of the robot, the crawling motion is achieved by multiple continuous postures. The structure and production process of Micro-soft crawling robot is introduced, and the quasi-static force balance equation is established. Comparing Abaqus finite element simulation and experimental results, the motion characteristics of the robot are analyzed, such as bending deformation, contact surface friction, crawling step length and steering. The speed model of robot crawling action is established, and the influence of the frequency and amplitude of control signal is analyzed. The path planning control of the crawling robot is carried out. The experimental and simulation results show that the robot can realize the crawling motion in any direction in the $x y$ plane, and this work may lay a foundation for further study of the motion characteristics and laws of the magnetic control micro-robot.
\end{abstract}

Key words: cable-less driven; micro-soft robot; magnetic elastic composite; crawling; magnetic control

\section{0 前言}

微型机器人作为一门涉及材料、机械、通信、

\footnotetext{
* 国家重点研发计划资助项目(2017YFB1302100)。20191010 收到初稿, 20200310 收到修改稿
}

生物医疗等多领域的新兴前沿学科，近些年来受到 广泛关注。微型机器人体积小, 能在普通机器人无 法进入的狭小复杂空间内工作 ${ }^{[1-3]}$, 在医疗、军事国 防、生物工程、微流控技术及微制造等领域有着广 阔的应用前景。

由于微型机器人体积小, 很难实现微型机器人 
内置能量供给与驱动, 所以能源供给与驱动是微型 机器人需解决的首要问题。无缆驱动可减小机器人 体积, 更好地满足微小作业的工作环境, 因此无缆 化是微型机器人发展的必然趋势。目前科研工作者 提出了利用微生物 ${ }^{[4]}$ 、化学反应 ${ }^{[5]}$ 以及磁场 ${ }^{[6-9]}$ 等方 案对其进行驱动和控制。HWANG 等 ${ }^{[10]}$ 利用电场产 生的电渗透力推动微型螺旋游泳微型机器人游动, 游泳速度达到了 $1.8 \mathrm{~mm} / \mathrm{s}$ 。哈佛大学的 WEHNER 等 ${ }^{[11]}$ 设计了章鱼软体微型机器人, 该微型机器人无 任何刚性材料, 利用过氧化氢分解的化学反应释放 气体实现肢体运动。卡内基梅隆大学的 EDWARDS 等 ${ }^{[12]}$ 用细菌与聚苯乙烯微球结合成生物混合微型 机器人, 在空间三维尺度上研究了细菌微球的作用 力、扭矩及移动规律。上述无缆驱动方法均能实现 微型机器人的预期动作, 但将其技术应用到医疗或 生物工程中还具有很大的挑战。例如, 压电式驱动 需要较高的电压, 而细菌驱动需保持细胞的低毒性 等, 这些控制条件限制了微型机器人的应用范围。 考虑到体内或组织工程环境的安全性和生物相容 性, 外磁场驱动方式更适用于无缆微型机器人的驱 动和控制。ZHANG 等 ${ }^{[13]}$ 提出了一种利用旋转均匀 磁场驱动的毫米级游动机器人, 建立数学模型对机 器人的偏转和磁化曲线之间的关系进行描述。CHOI 等 ${ }^{[14]}$ 提出了一种基于亥姆霍兹和麦克斯韦线圈的 微型机器人的二维驱动方法, 通过控制线圈系统续 电流, 使机器人沿理想的路径运动。由于微型机器 人易受到外界液体、噪声、摩擦 ${ }^{[15]}$ 以及边界效应等 多种干扰, 目前对微型机器人的研究存在的问题主 要是理论或仿真模型较为简单, 导致其控制精度和 重复性还不能达到实际应用的要求。

本文基于磁弹性复合材料设计了一种新型磁控 微型软体爬行机器人, 搭建三维亥姆霍兹线圈来实 现对机器人的控制, 利用 Abaqus 有限元软件建立了 机器人仿真模型, 并将仿真结果和试验数据进行对 比分析。本文利用理论研究、有限元分析以及试验 探究相结合, 对机器人的姿态运动学进行了深入分 析, 更为精确地描述了微型软体机器人的各种运动 特性和本质规律, 在此基础上, 设计相应的控制策 略实现微型机器人的爬行和旋转等动作, 进一步完 成微型软体机器人的精确控制。

\section{1 试验系统与爬行机器人结构设计}

\section{1 试验系统}

微型机器人试验控制系统如图 1 所示, 采用三
维亥姆霍兹线圈来构建空间任意方向的均匀磁场, 通过均匀磁场来控制基于磁弹复合材料的微型软体 爬行机器人的各种动作姿态。

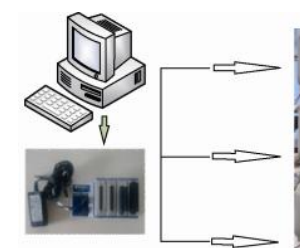

IPC+LabView

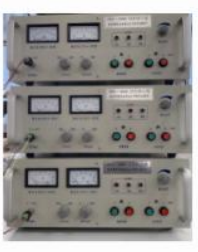

功率放大器

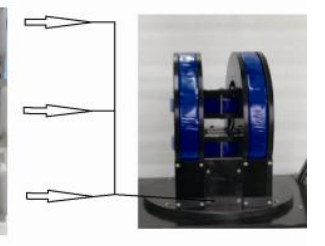

亥姆霍兹线圈
图 1 试验控制系统

系统由工控机(IPC)、Labview CompactDAQ (Laboratory Virtual Instrumentation Engineering Workbench, NI cDAQ-9174)、功率放大器及三维亥 姆霍兹线圈组成。Labview 模块输出三路电压信号, 经功率放大器转化为三对正交亥姆霍兹线圈的控制 电流, 从而产生空间任意方向的均匀磁场, 构建微 型软体爬行机器人各种运动姿态所需的磁场环境。

建立空间磁场球坐标系 $(B, \gamma, \varphi)$, 如图 2 所示, $B$ 为外加磁场的磁感应强度, 空间均匀磁感应强度 $B$ 与 $x y$ 平面的夹角为 $\gamma$, 与 $x z$ 平面的夹角为 $\varphi$, 其 中 $\gamma$ 和 $\varphi$ 逆时针为正。微型机器人放置于磁场坐标 系的 $x y$ 平面上, 通过三维亥姆霍兹线圈产生的均匀 磁场实现对微型机器人的运动姿态的控制。

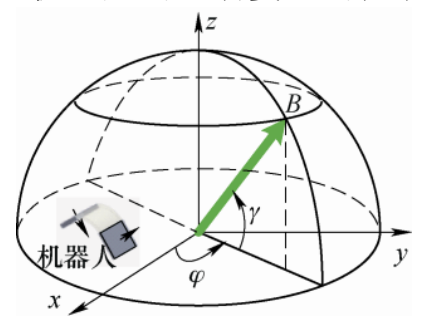

图 2 空间磁场球坐标系

\section{2 微型软体爬行机器人的结构设计}

爬行机器人结构如图 3 所示, 其头、尾部分由 两个大小相同磁化方向相反的磁性材料制成; 中间 部分采用弹性材料, 对头尾两部分进行连接, 后简 称此部分为关节。当外部磁场为零时, 机器人处于 自然平放状态; 当外部磁场不为零时, 机器人受磁

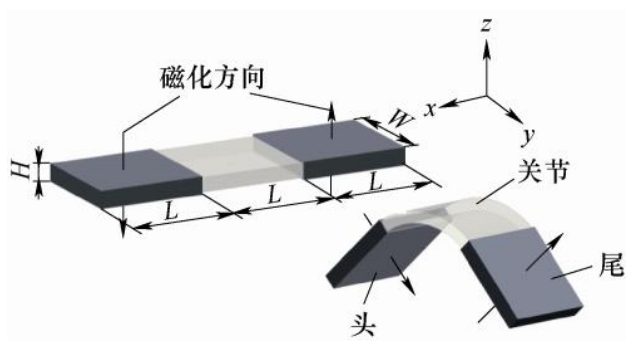

图 3 机器人结构示意图 
力矩、重力、支撑力、摩擦力等外力作用, 身体弯 曲为拱形。机器人的几何参数如表 1 所示。

表 1 机器人各部分尺寸(mm)

\begin{tabular}{cccc}
\hline \multirow{2}{*}{ 部位 } & \multicolumn{3}{c}{ 参数 } \\
\cline { 2 - 4 } & 长 & 宽 & 高 \\
\hline 头/尾/关节 & 3 & 3 & 0.5 \\
\hline
\end{tabular}

微型软体爬行机器人的制作流程如图 4 所示, 首先, 利用微米级高精度光固化 3D 打印机(Formlabs Form1)制作模具, 模具包含机器人头尾磁块制作和 整体制作两个模块; 然后将高弹性铂催化硅橡胶 (Smooth-On Ecoflex 00-10)材料与高磁能积及高剩 磁的磁性粒子(YMM-E-15-7, NdFeB)按照体积比 $1: 0.15$ 均匀混合, 再将混合物倒入制作磁块的凹槽 内; 待混合物凝固后将其放入能产均匀磁场的充磁 机(ME-1225)进行磁化; 将磁化后的小磁块取出, 头、尾以相反的方向放入机器人整体制作模块中, 最后利用硅橡胶填充中间关节部分, 对头尾部分进 行柔性连接, 待材料完全凝固后, 取出微型软体爬 行机器人。

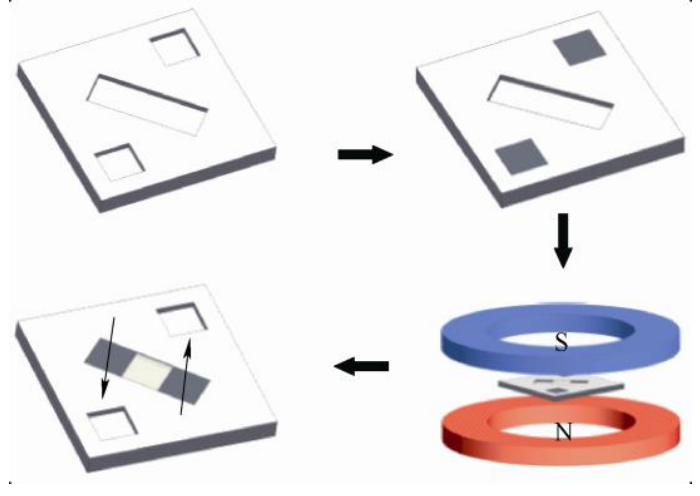

图 4 微型软体爬行机器人制作流程

\section{2 受力分析}

磁性材料在均匀磁场中受到磁力矩作用, 可表 示为

$$
\tau=M B V \sin \alpha
$$

式中, $M$ 为磁性材料的磁化强度; $B$ 为外加磁场的 磁感应强度; $V$ 为磁性材料体积; $\alpha$ 为磁化方向与 外加磁场方向的夹角。

微型爬行机器人的运动特性由其材料特性、几 何结构及外加磁场决定。由于机器人质量非常小, 因此在外部力和力矩作用下能快速达到平衡状态, 本文利用准静态分析方法来分析机器人的各阶段的 运动状态。
当外部施加均匀磁场时, 机器人的受力分析如 图 5 所示, 外部磁感应强度 $B$ 与 $x y$ 平面成 $\gamma$ 角, 由 式(1), 此时机器人首尾受到方向不同的磁力矩 $\tau_{1}$ 与 $\tau_{2}$, 使得机器人关节部分呈弯曲状态, 其中 $h$ 表示 机器人弯曲最高点与底面的纵向位移, $a 、 b$ 分别为 首尾部分与底面的接触点, $c 、 d$ 分别为关节与头部 和尾部的连接点。

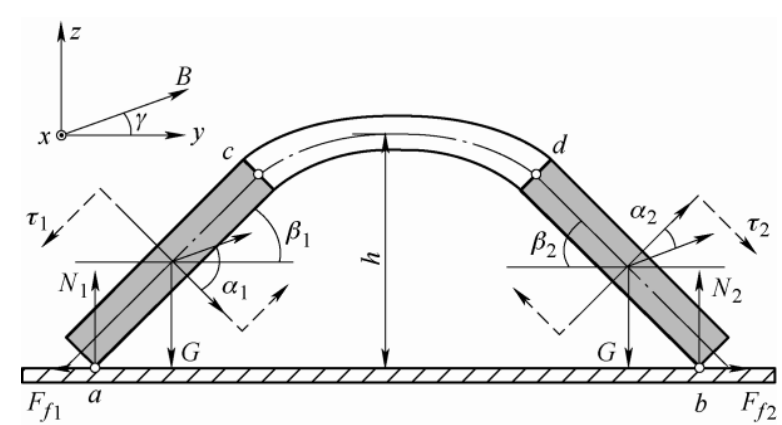

图 5 机器人受力示意图

机器人首尾部分磁力矩可表示为

$$
\left\{\begin{array}{l}
\tau_{1}=M B V \sin \alpha_{1} \\
\tau_{2}=M B V \sin \alpha_{2}
\end{array}\right.
$$

$\alpha_{1}$ 与 $\alpha_{2}$ 几何关系如下

$$
\left\{\begin{array}{l}
\frac{\pi}{2}+\gamma-\alpha_{1}-\beta_{1}=0 \\
\frac{\pi}{2}-\gamma-\alpha_{2}-\beta_{2}=0
\end{array}\right.
$$

式中, $\beta_{1}$ 与 $\beta_{2}$ 分别为机器人首尾与水平面 $x y$ 的夹角。

由于关节部分采用密度较小的弹性材料制成, 为简化仿真模型, 忽略此部分质量, 本文仅考虑机 器人首尾两部分的重力, 均为 $G$ 。以支撑点 $a$ 为参 考点建立力矩平衡方程

$$
\begin{gathered}
\left(\frac{3}{2} G-N_{2}\right) L \cos \beta_{1}+\left(\frac{1}{2} G-N_{2}\right) L \cos \beta_{2}+ \\
\left(G-N_{2}\right) w_{x L}-\tau_{1}+\tau_{2}=0
\end{gathered}
$$

式中, $N_{1} 、 N_{2}$ 分别为机器人首尾部分在支撑点 $a 、 b$ 处所受的支撑力。 $w_{x L}$ 表示关节部分弯曲后在 $x$ 轴方 向的距离。

在 $x 、 y$ 方向的力平衡方程如下

$$
\begin{gathered}
N_{1}+N_{2}-2 G=0 \\
F_{f 1}=F_{f 2}
\end{gathered}
$$

式中, $F_{f 1} 、 F_{f 2}$ 分别表示支撑点 $a 、 b$ 处的摩擦力。

如图 6 所示, 在磁力矩作用下，机器人关节部 分弯曲为拱形状态。该部分可近似梁的弯曲理论进 行分析, 沿梁轴线上任意一点为 $s, \beta$ 表示该点的角 位移, $w_{x}(s)$ 表示该点在 $x$ 方向与 $c$ 点的相对距离, 
$w_{y}(s)$ 即为其在 $y$ 方向与 $c$ 点相对距离。根据挠曲轴 理论可得到如下方程

$$
\begin{aligned}
& w_{x}(s)=\int_{0}^{s} \cos \beta \mathrm{d} s \\
& w_{y}(s)=\int_{0}^{s} \sin \beta \mathrm{d} s
\end{aligned}
$$

式中, $s$ 的取值范围为 $[0, L]$ 。

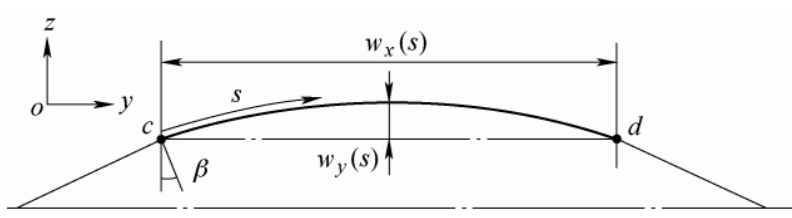

图 6 关节部分的梁弯曲分析

弯曲状态下, 关节处机器人所受的弯矩为 $Q$, 由欧拉伯努利梁理论可得

$$
\frac{\mathrm{d} \beta}{\mathrm{d} s}=\frac{Q}{E I}
$$

式中, $E I$ 为关节部分的弯曲刚度。

同时, 由剪切力与弯矩的关系可得

$$
\frac{\mathrm{d} Q}{\mathrm{~d} s}=\left(N_{1}-G\right) \cos \beta
$$

\section{3 运动特性的仿真与试验分析}

\section{1 微型爬行机器人的力学特性分析}

为探究空间磁场对微型机器人运动特性的影 响, 本文利用 Abaqus 建立仿真模型, 通过仿真和试 验分别对比分析了爬行机器人在对称和非对称状态 下的力学特性。

当外部磁场平行于 $y$ 轴(即 $\gamma=0$ ) 时, 机器人首 尾所受到的磁力矩 $\tau_{1}$ 与 $\tau_{2}$ 大小相同、方向相反, 此 时机器人首尾处于对称状态。随着磁感应强度的增 加, 机器人的弯曲程度也逐渐增大。试验与仿真对 比示意图如图 7 所示, 其中, 微型机器人关节处的 弹性模量为 $0.1 \mathrm{MPa}^{[16]}$, 泊松比为 0.33 , 首尾部分 磁弹复合材料的密度为 $1.86 \mathrm{~g} / \mathrm{cm}^{3}$ 。



图 7 对称状态下磁感应强度对机器人姿态的影响
磁感应强度 $B$ 在 $[0,100 \mathrm{Gs}$ ]范围内, 分别进行 试验和仿真分析, 研究了机器人首尾部分与水平面 的夹角 $\beta_{1} 、 \beta_{2}$, 机器人弯曲纵向位移 $h$ 与磁感应强 度 $B$ 的关系, 试验结果与仿真数据对比分析如图 8 和图 9 所示。结果表明, 在磁场角度 $\gamma=0$ 时, 机器 人关节弯曲处于对称平衡状态, 机器人首尾与水平 面的夹角 $\beta_{1}$ 与 $\beta_{2}$ 大小相等, 同时它们随着磁感应强 度 $B$ 增大而逐渐增大, 机器人弯曲纵向位移 $h$ 随着 磁感应强度 $B$ 增大而逐渐增大, 试验数据与仿真结 果基本一致。

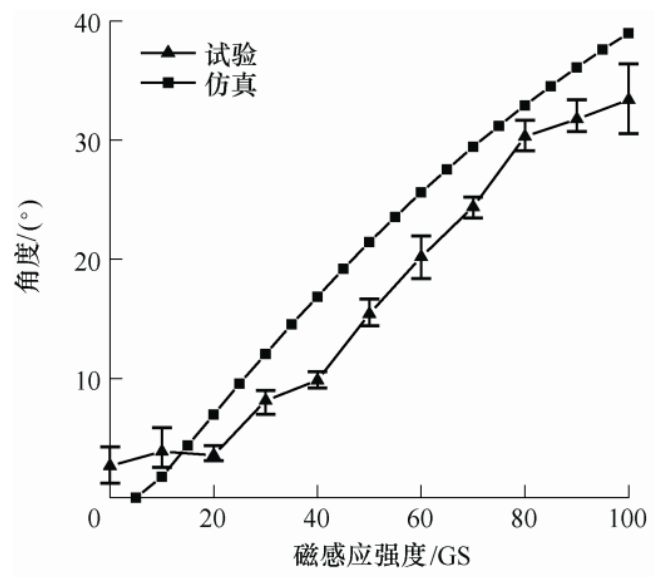

图 $8 \beta_{1} 、 \beta_{2}$ 与磁感应强度 $B$ 的关系

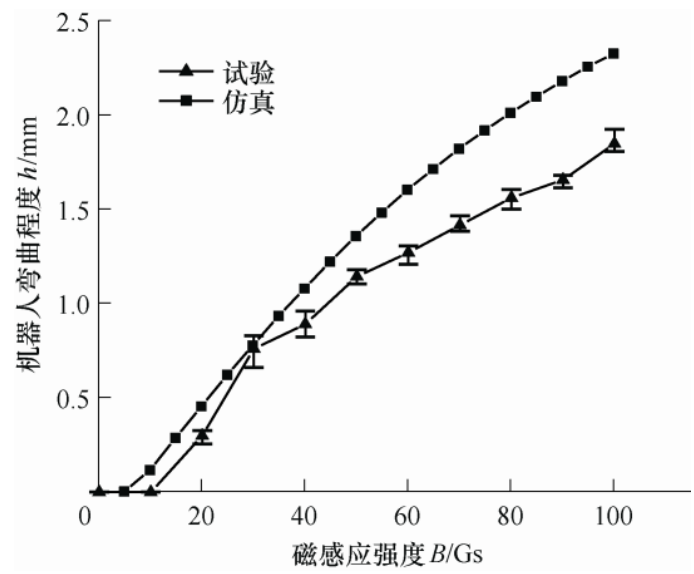

图 9 弯曲纵向位移 $h$ 与磁感应强度 $B$ 的关系

当外部磁场方向与 $y$ 轴成一定夹角时 (即 $\gamma \neq 0)$, 由于机器人所受磁力矩 $\tau_{1}$ 与 $\tau_{2}$ 大小不同、方向相反, 机器人首尾处于非对称状态。如图 10 所示, 图 10a 为当磁感应强度 $B=75 \mathrm{Gs}, \gamma=20^{\circ}$ 时, 机器人首尾分 别在支撑点 $a 、 b$ 处的支撑力 $N_{1} 、 N_{2}$ 的仿真结果, 可以看出在非对称条件下 ( $\gamma>0$ 时), $a$ 点支撑力 $N_{1}$ 大于 $b$ 点处的支撑力 $N_{2}$; 图 $10 \mathrm{~b}$ 为磁感应强度 $B$ 在 $[0,100 \mathrm{Gs}]$ 范围内, $\gamma=20^{\circ}$ 时, 支撑力 $N_{1}$ 与 $N_{2}$ 的仿 真结果, 由图可知, 机器人头部 $a$ 点所受支撑力 $N_{1}$ 随着磁感应强度的增大不断增大, 机器人尾部 $b$ 点 
所受支撑力 $N_{2}$ 随磁感应强度增大不断减小, 当 $B>95 \mathrm{Gs}$ 时, $b$ 点处于悬空抬起状态, 此时 $a$ 点支 撑力 $N_{1}$ 为机器人首尾两部分重力之和 $2 G, b$ 点支 撑力 $N_{2}$ 为 0 。

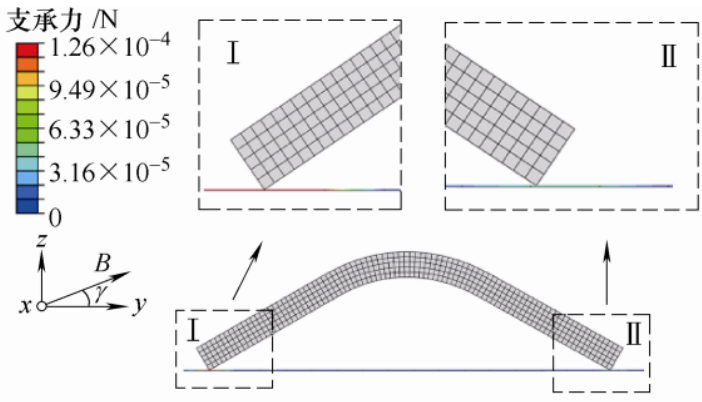

(a) 非对称状态下仿真结果 $\left(B=75 \mathrm{Gs}, \gamma=20^{\circ}\right)$

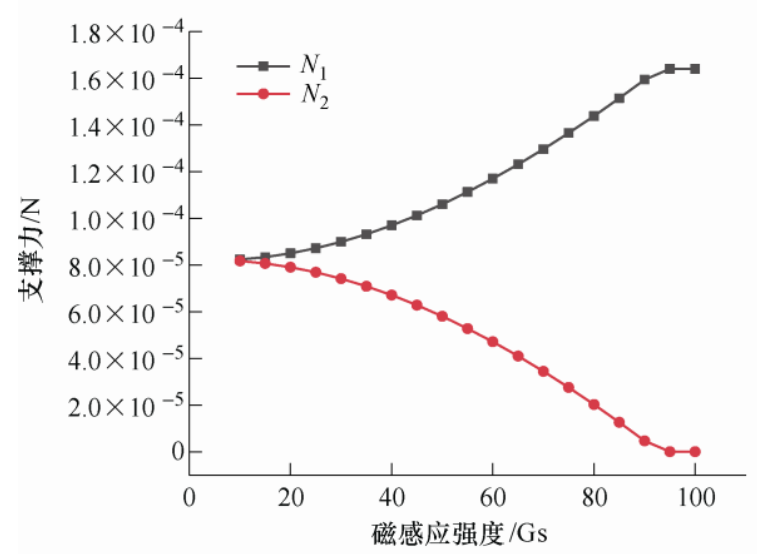

(b) 非对称状态下 $N_{1} 、 N_{2}$ 与磁场角度 $B$ 的关系 $\left(\gamma=20^{\circ}\right)$

图 10 非对称状态下仿真结果

在非对称状态条件下, 磁感应强度 $B$ 在 $[0$, $100 \mathrm{Gs}$ 范围内, $\gamma=20^{\circ}$ 时, $\beta_{1} 、 \beta_{2}$ 与磁感应强度的 关系结果如图 11 所示, $\beta_{1} 、 \beta_{2}$ 随磁感应强度增大而 逐渐增大, 当 $B>95 \mathrm{Gs}$ 时, 机器人尾部被抬起, $\beta_{1}$ 曲线的斜率快速增大, 而 $\beta_{2}$ 曲线的斜率逐渐变小, 试验和仿真结果基本一致。

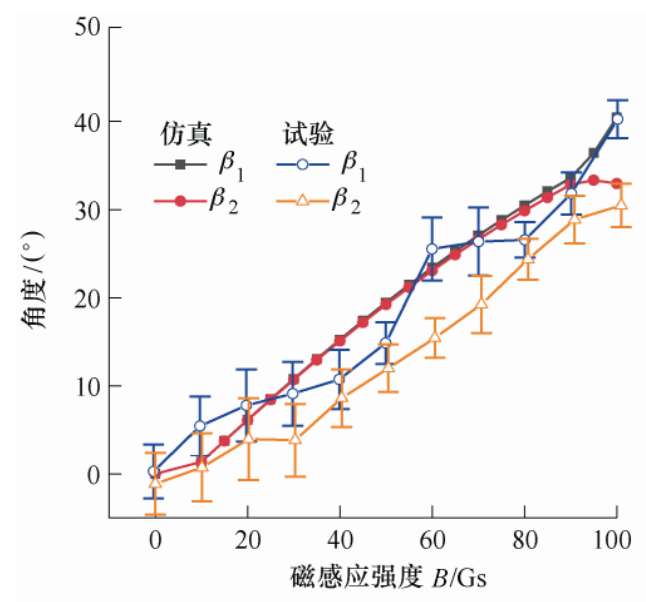

图 11 非对称状态 $\beta_{1} 、 \beta_{2}$ 与磁场角度 $\gamma$ 的关系 $\left(\gamma=20^{\circ}\right)$

\section{2 微型爬行机器人的运动步长分析}

机器人在运动过程中, 底面接触点 $a 、 b$ 处受非 线性摩擦影响, 当机器人与底面相对静止时, 其摩 擦力为静摩擦力, 其大小随该方向上的其他合力而 改变, 在即将发生相对位移的瞬间, 此时摩擦力为 最大静摩擦力, 最大静摩擦因数 $\mu_{\mathrm{m}}$; 当机器人与底 面有相对滑动时, 其摩擦力为滑动摩擦力, 滑动摩 擦因数为 $\mu_{0}$, 其中, $\mu_{0}<\mu_{\mathrm{m}}$ 。

摩擦力与支撑力的关系如下

$$
F_{f}= \begin{cases}\mu_{m} N & u=0 \\ \mu_{0} N & u \neq 0\end{cases}
$$

式中, $u$ 表示与底面的相对位移。

在非对称条件下, 由图 $10 \mathrm{~b}$ 和图 11 可知, 随磁 感应强度的不断增大, 机器人的弯曲程度逐渐增大, 在 $a 、 b$ 点处所受到的支撑力也有所不同。由式(11) 可知, $a$ 点处的最大静摩擦力远大于 $b$ 点处的最大 静摩擦力, 因此随着磁感应强度的不断增加, 机器 人弹性形变会克服 $b$ 点最大静摩擦力而产生滑动。 依据准静态分析式(6), 利用准静态方程任意时刻机 器人头部 $a$ 点的所受到的摩擦力 $F_{f 1}$ 与尾部 $b$ 点摩擦 力 $F_{f 2}$ 相等, 即 $a$ 点处受到的静摩擦力与 $b$ 点处的滑 动摩擦力平衡, 其运动现象表现为 $a$ 点与底面相对 静止, $b$ 点相对滑动。

在此基础上, 进一步完成爬行机器人的一个完 整单步爬行动作, 此过程包含一个连续的收缩运动 和伸展运动, 其原理如图 12 所示。首先对微型爬行 机器人施加外部磁场 $B$, 与 $x y$ 平面夹角为 $\gamma$, 其大 小由零增加到拟定值, 机器人由自然平放状态 1 逐 渐变弯曲到状态 2 , 当外部磁场达到最大值时机器 人达到最大弯曲状态 3 , 完成收缩运动, 该过程中 头部 $a$ 点与底面相对静止, 尾部 $b$ 点与底面相对滑 动; 伸展运动与收缩运动特性相反, 对微型爬行机 器人施加外部磁场 $B$, 与 $x y$ 平面夹角为 $-\gamma$, 磁感应 强度由拟定值逐渐减小至零, 机器人由弯曲状态 4 经状态 5 逐渐变为自然平放状态 6 , 该过程中 $b$ 点 的支撑力大于 $a$ 点的支撑力, 因此 $b$ 点处受到的静 摩擦力与 $a$ 点处的滑动摩擦力平衡, 即 $b$ 点与底面 相对静止, 头部 $a$ 点相对滑动。

定义机器人单次爬行动作的位移为步长 $s_{t}$

$$
s_{t}=3 L-L\left(\cos \beta_{1 \text { max }}+\cos \beta_{2 \max }\right)-w_{x L}
$$

式中, $\beta_{1 \max } 、 \beta_{2 \max }$ 分别为在外部磁感应强度 $B$ 作用 下首尾与水平面 $x y$ 的最大夹角。

微型机器人的前进、后退动作是由多个爬行动 作的积累结果, 本文利用 Abaqus 有限元仿真与试验 


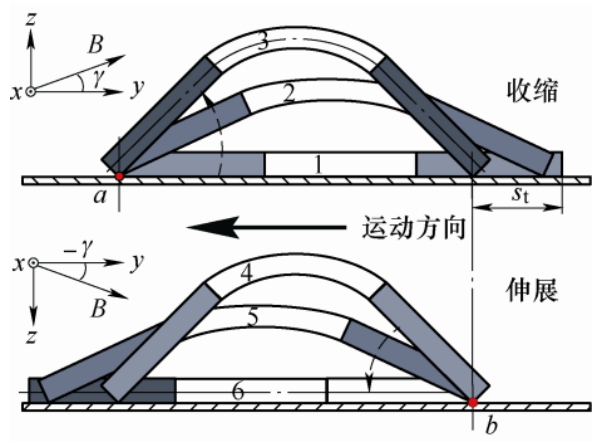

图 12 机器人完整的单步爬行运动过程

进行了对比, 其示意图如图 13 所示, 进一步分析磁 感应强度 $B$ 与机器人爬行步长 $s_{t}$ 的关系, 试验与仿 真数据如图 14 所示。

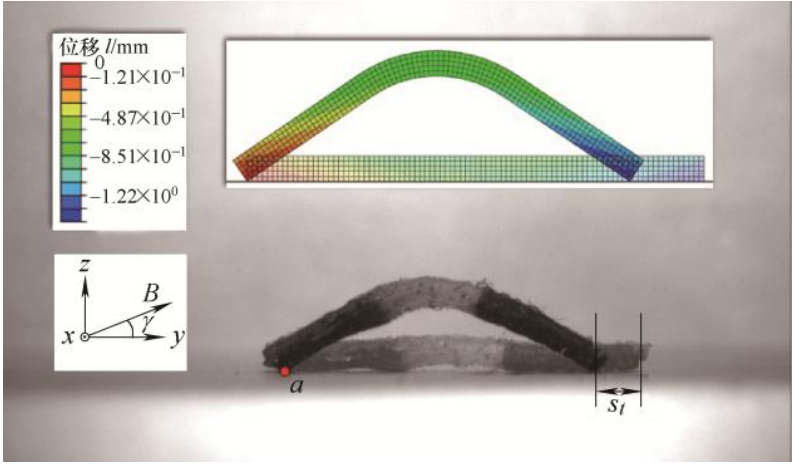

图 13 步长分析试验及仿真结果

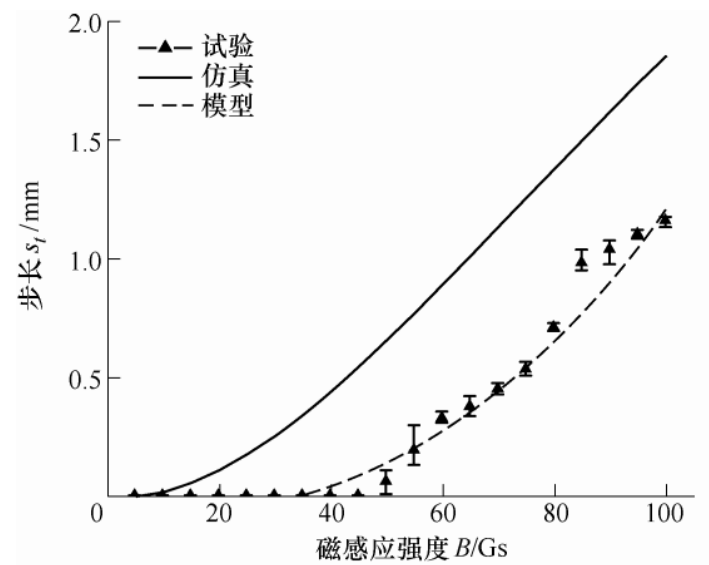

图 14 步长 $s_{\mathrm{t}}$ 与磁感应强度 $B$ 的关系

由图 14 可知, 微型机器人爬行动作的步长 $s_{t}$ 随着磁感应强度 $B$ 的增大逐渐增大, 试验数据与仿 真结果趋势基本一致。拟合磁感应强度与步长的试 验数据, 建立微型机器人爬行动作步长模型为

$$
s_{t}=0.0002 B^{2}-0.0087 B+0.0661
$$

当对微型机器人重复施加频率为 $f$ 的单步爬行 磁场控制信号时, 机器人可实现沿某一方向以一定 速度爬行, 其爬行速度的模型可表示为

$$
v=\left(0.0002 B^{2}-0.0087 B+0.0661\right) f
$$

\section{3 微型爬行机器人的转向运动特性分析}

微型爬行机器人转向动作的控制是通过改变空 间磁场绕 $z$ 轴的旋转角度 $\varphi$ 实现, 其原理如图 15 所 示, 当机器人弯曲成拱形时, 机器人自身磁化方向 与外加磁场方向存在一定的偏角, 因此当外部磁场 绕 $z$ 轴旋转时, 形成以 $z$ 轴为轴心的旋转转矩, 爬 行机器人也随着 $\varphi$ 值的改变而产生相应的转动。

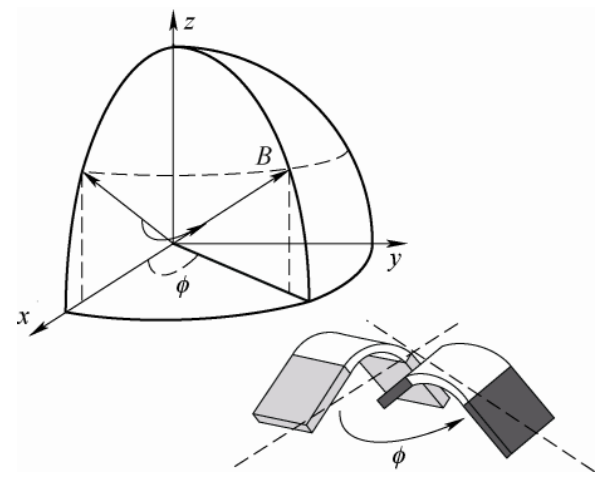

图 15 机器人转向角 $\varphi$ 的控制原理

定义爬行机器人转向运动的模型为

$$
\varphi_{r}(t)=k_{\varphi} t
$$

式中， $k_{\varphi}$ 表示转向角速度。

由图 16 可知, 微型机器人转向运动的试验和转 向运动模型数据基本一致, 转向角与时间成线性关 系, 其斜率为转向角速度 $k_{\varphi}$ 。

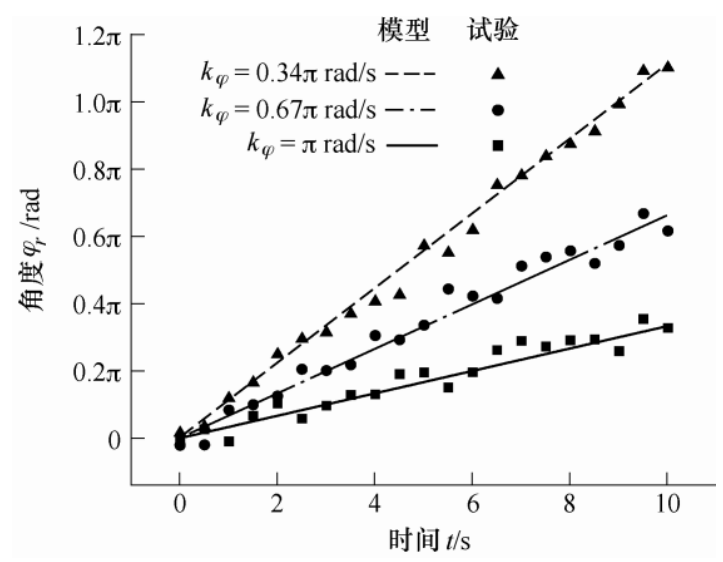

图 16 爬行机器人转向试验

\section{4 路径规划与控制}

\section{1 激励信号}

根据空间磁场球坐标系 $(B, \gamma, \varphi)$, 对机器人施 加周期性变化磁场, 该信号由亥姆霍兹线圈 $x y z$ 三 路驱动信号合成, 通过改变单周期输入信号 $B 、 \gamma$ 、 $\varphi$ 参数及频率可实现对机器人运动方向及速度的控 制。如图 17 所示, 机器人爬行频率为 $1 \mathrm{~Hz}$, 最大 
磁感应强度 $B=100 \mathrm{Gs}, \gamma$ 在 $\left[-20^{\circ}, 20^{\circ}\right]$ 范围内, $\varphi=30^{\circ}$ 时所对应的 $x y z$ 三路电压控制信号。

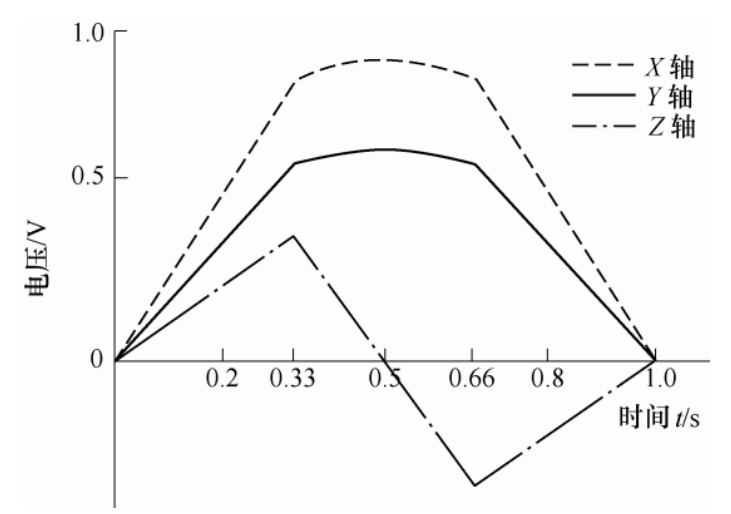

图 $17 x y z$ 三路电压控制信号

在上述驱动信号下, 爬行机器人在一个运动周 期内的姿态变化特性如图 18 所示, 改变外部磁场方 向角 $\varphi$, 可完成爬行机器人在 $x y$ 平面任意方向的爬 行动作。

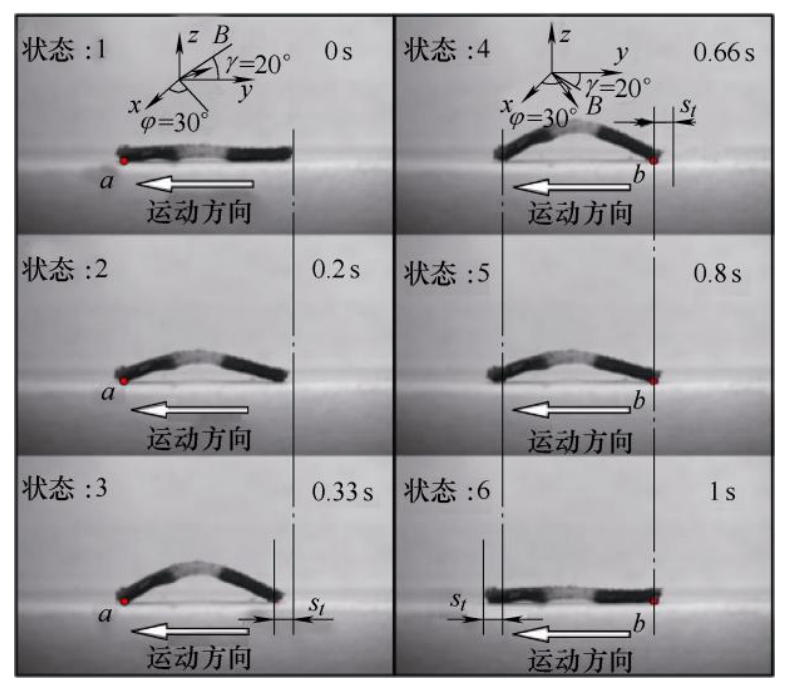

图 $181 \mathrm{~Hz}$ 条件下机器人单周期内爬行姿态

\section{2 激励信号频率对运动速度影响}

在爬行运动中, 激励信号的频率直接关系到爬 行机器人的迈步频率, 从而影响到爬行机器人运动 速度。分别取磁感应强度为 60 Gs、 80 Gs、 100 Gs, 激励信号频率 $f$ 在 $[0,20 \mathrm{~Hz}]$ 范围内进行相关试验, 试验结果如图 19 所示。

由图 19 可知, 当磁感应强度一定, 仅改变激励 信号的频率, 机器人的运动速度 $v$ 随着驱动信号频 率 $f$ 增大线性增大, 试验数据基本符合的爬行机器 人运动速度模型。

\section{3 激励信号幅值对运动速度影响}

步长决定了爬行机器人单个运动环节的行进距 离, 输入信号的最大磁感应强度直接影响爬向机器人 的步长, 分别取激励信号频率为 $3 \mathrm{~Hz} 、 4 \mathrm{~Hz}$ 和 $5 \mathrm{~Hz}$,

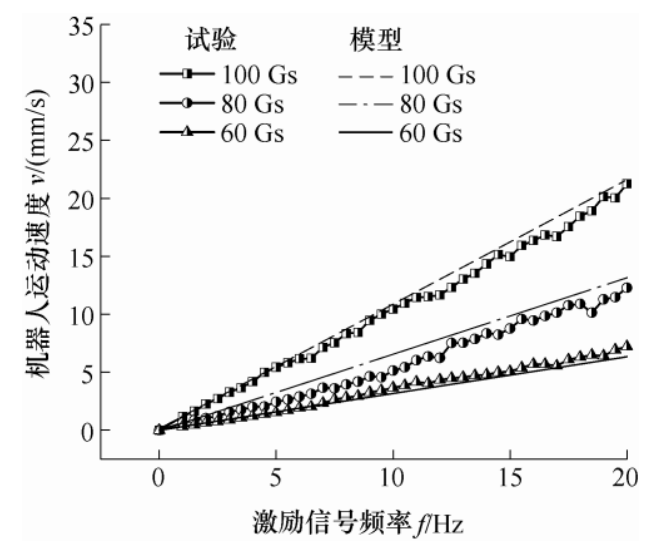

图 19 激励信号频率 $f$ 与机器人运动速度 $v$ 的关系

探究输入信号的最大磁感应强度 $B$ 与机器人运动速 度 $v$ 之间的关系, 试验结果如图 20 所示。

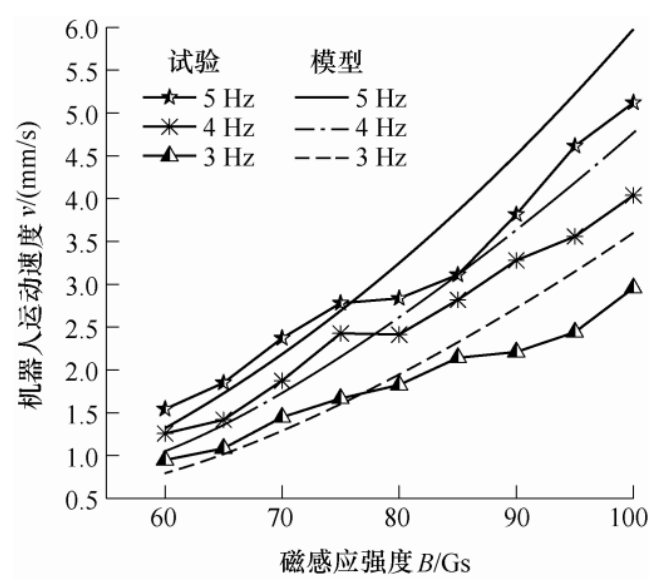

图 20 磁感应强度 $B$ 与机器人运动速度 $v$ 的关系

由图 20 可知, 当激励信号频率一定, 微型爬行 机器人的运动速度 $v$ 随着磁感应强度 $B$ 的增大而逐 渐增大, 试验数据基本符合的爬行机器人运动速度 模型。

\section{4 路径规划控制试验}

设计驱动信号频率 $f=3 \mathrm{~Hz}$, 最大磁感应强度 $B$ 为 $100 \mathrm{Gs}$, 磁场角度 $\gamma$ 在 $\left[-20^{\circ}, 20^{\circ}\right]$ 范围时, 爬行 机器人在 $x y$ 平面的规划运动路线如图 21 所示。



图 21 路线规划 
根据规划路线设计相应的控制信号如图 22 所 示, 其中转向角 $\varphi$ 的变化曲线如图 22a 所示, 取时 间 $t$ 在 $[3 \mathrm{~s}, 4 \mathrm{~s}]$ 范围时, 亥姆霍兹线圈 $x y z$ 三路驱动 信号的变化曲线如图 22b 所示。

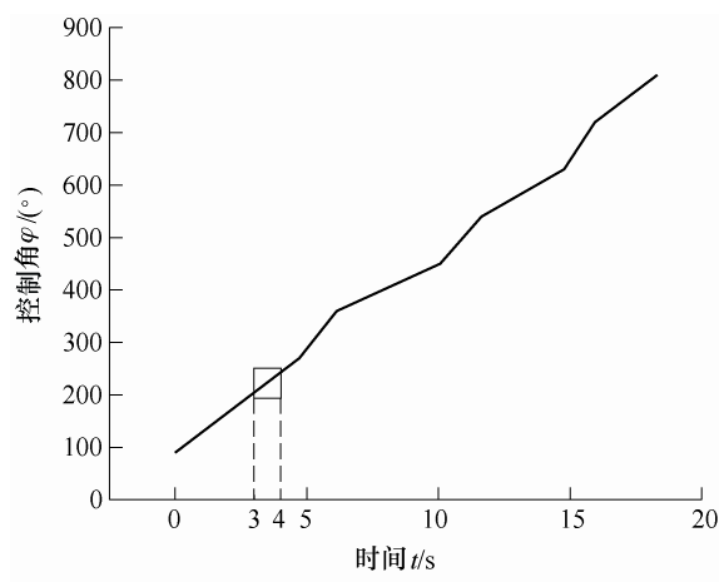

(a) 机器人控制角 $\varphi$ 变化曲线



(b) $t$ 在 $[3 \mathrm{~s}, 4 \mathrm{~s}]$ 范围时 $x y z$ 三路控制信号的变化曲线

图 22 机器人轨迹控制输入信号

以上述驱动激励信号作为爬行机器人的输入控 制信号, 并记录试验结果如图 23 所示, 由图 23 可 知, 该试验路径与规划路线基本重合, 能完成在 $x y$ 平面内的任意规划路线。

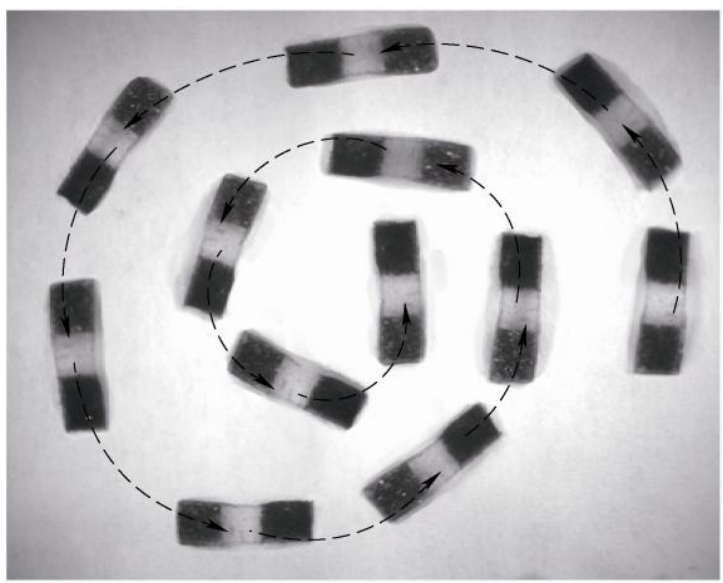

图 23 试验控制路径

\section{5 结论}

在详细分析亥姆霍兹线圈产生磁场理论和建立 磁性物体在均匀磁场受力平衡方程的基础上, 设计 了一种微型软体爬行机器人, 并对其运动特性进行 试验和仿真分析, 结论如下。

(1) 建立了微型软体爬行机器人的仿真模型, 通过仿真分析与试验验证, 得到了对称状态和非对 称状态下机器人弯曲程度 $h$ 、首尾支撑力 $N_{1} 、 N_{2}$, 水平面夹角 $\beta_{1} 、 \beta_{2}$ 与磁感应强度 $B$ 和磁场角度 $\gamma$ 的 动态关系。

(2) 分析微型机器人实现机器人爬行运动原 理, 并通过仿真描述了磁感应强度与机器人步长的 动态关系，进一步通过施加不同频率激励信号、磁 感应强度幅值等试验验证了爬行机器人的运动速 度模型, 在此基础上实现了爬行机器人的路径规划 控制。

(3) 试验数据与仿真分析结果基本一致, 但仍 存在一定的偏差, 主要原因为: 微型机器人的制作 工艺精度不够, 实物与理论设计有一定客观偏差, 此外, 机器人在位姿变化运动过程中, 弹性材料在 释放能量时存在振荡现象, 准静态分析不能完全的 描述爬行机器人的动态运动过程。

本文通过仿真分析与试验验证了微型软体爬行 机器人理论设计的合理性以及实际控制操作的可行 性, 研究结果对进一步推动磁控无缆式微型机器人 的研究，具有指导意义和参考价值。

\section{参 考 文 献}

[1] DILLER E, SITTI M. Three-dimensional programmable assembly by untethered magnetic robotic micro-grippers[J]. Adv. Funct. Mater., 2014, 24: 4397-4404.

[2] 张永顺, 周华涛, 张林霞, 等. 一种新型双半球形胶囊 机器人[J]. 机械工程学报, 2017, 53(15): 110-118.

ZHANG Yongshun, ZHOU Huatao, ZHANG Linxia, et al.

A new kind of dual hemisphere capsule robot[J]. Journal of Mechanical Engineering，2017，53(15): 110-118.

[3] 王田苗, 郝雨飞, 杨兴帮, 等. 软体机器人: 结构、驱动、 传感与控制[J]. 机械工程学报，2017，53(13): 1-13.

WANG Tianmiao, HAO Yufei, YANG Xingbang, et al. Soft robotics: Structure, actuation, sensing and control[J]. Journal of Mechanical Engineering, 2017，53(13): 1-13.

[4] WILLIAMS B J, ANAND S V, RAJAGOPALAN J, et al. A self-propelled biohybrid swimmer at low Reynolds 
number[J]. Nat. Commun., 2014, 5: 3081.

[5] SOLOVEV A A, XI W, GRACIAS D H, et al. Self-propelled nanotools[J]. ACS Nano, 2012, 6(2): 1751-1756.

[6] MAHONEY A W, NELSON N D, PEYER K E, et al. Behavior of rotating magnetic microrobots above the step-out frequency with application to control of multimicrorobot systems[J]. Appl. Phys. Lett., 2014, 104(4): 144101 .

[7] 张永顺, 孙颖, 杜春雨, 等. 胶囊机器人弯曲环境通过 性与磁矢量控制[J]. 机械工程学报, 2014, 50(5): 26-32. ZHANG Yongshun, SUN Ying, DU Chunyu, et al. Trafficability characteristic and magnetic vector control of a capsule robot in bending environment[J]. Journal of Mechanical Engineering, 2014, 50(5): 26-32.

[8] HU Wenqi, ZHAN L G, MASSIMO M, et al. Small-scale soft-bodied robot with multimodal locomotion[J]. Nature, 2018, 554: 81-85.

[9] KUMAR N, VERMA V, BEHERA L. Magnetic navigation and tracking of multiple ferromagnetic microrobots inside an arterial phantom setup for MRI guided drug therapy[J]. Biocybernetics and Biomedical Engineering, 2017, 37(3): 347-356.

[10] HWANG G, BRAIVE R, COURAUD L, et al. Electroosmotic propulsion of helical nanobelt swimmers[J]. Int. J. Robot Res., 2011， 30(7): 806-819.

[11] WEHNER M, TRUBY R L, FITZGERALD D J, et al. An integrated design and fabrication strategy for entirely soft, autonomous robots[J]. Nature, 2016, 536: 451-455.

[12] EDWARDS M R, CARLSEN R W, SITTI M. Near and far-wall effects on the three-dimensional motion of bacteria-driven microbeads[J]. Appl. Phys. Lett., 2013, 102: 14701 .

[13] ZHANG J , DILLER E. Millimeter-scale magnetic swimmers using elastomeric undulations[C]// IEEE/RSJ International Conference on Intelligent Robots and Systems (IROS). Hamburg: IEEE，2015: 1706-1711.

[14] CHOI H, CHOI J, JANG G, et al. Two-dimensional actuation of a microrobot with a stationary two-pair coil system[J]. Smart Materials and Structures, 2009, 18: 055007.

[15] XIANG Hongbiao, TRKOV M, YU K. A stick-slip interactions model of soft-solid frictional contacts[J]. Journal of Dynamic Systems, Measurement, and Control, 2019, 141(4): 041015.

[16] PARK S, MONDAL K, TREADWAY R M, et al. Silicones for stretchable and durable soft devices: Beyond Sylgard-184[J]. ACS Appl. Mater. Interfaces, 2018, 10(13): 11261-11268.

作者简介: 向红标(通信作者), 男, 1982 年出生, 副教授。主要研究方 向为微型机器人, 伺服控制技术。

E-mail: xhb@tju.edu.cn

李岩, 男, 1995 年出生, 硕士研究生。主要研究方向为柔弹性复合材料、 微型机器人。

E-mail: 1521630658@qq.com 